\title{
Data mining of pediatric medulloblastoma microarray expression reveals a novel potential subdivision of the Group 4 molecular subgroup
}

\author{
ROSA ANGÉLICA CASTILLO-RODRÍGUEZ ${ }^{1,2}$, VÍCTOR MANUEL DÁVILA-BORJA ${ }^{1}$ \\ and SERGIO JUÁREZ-MÉNDEZ ${ }^{1}$
}

${ }^{1}$ Laboratory of Experimental Oncology; ${ }^{2}$ CONACyT, National Institute of Pediatrics, Mexico City 04530, Mexico

Received April 21, 2017; Accepted January 11, 2018

DOI: $10.3892 / \mathrm{ol} .2018 .8094$

\begin{abstract}
Medulloblastoma is the most common type of solid brain tumor in children. This type of embryonic tumor is highly heterogeneous and has been classified into 4 molecular subgroups based on their gene expression profiles: WNT, SHH, Group 3 (G3) and Group 4 (G4). WNT and SHH tumors exhibit the specific dysregulation of genes and pathways, whereas G3 and G4 tumors, two of the more frequent subtypes, are the least characterized. Thus, novel markers to aid in the diagnosis, prognosis and management of medulloblastoma are required. In the present study, microarray gene expression data was downloaded from the Gene Expression Omnibus database, including data from the 4 subgroups of medulloblastoma and healthy cerebellum tissue (CT). The data was utilized in an in silico analysis to characterize each subgroup at a transcriptomic level. Using Partek Genomics Suite software, the data were visualized via hierarchical clustering and principal component analysis. The differentially expressed genes were uploaded to the MetaCore portal to perform enrichment analysis using CT gene expression as baseline, with fold change thresholds of $<-5$ and $>5$ for differential expression. The data mining analysis of microarray gene expression data enabled the identification of a range of dysregulated molecules associated with each subgroup of medulloblastoma. G4 is the most heterogeneous subgroup, as no definitive pathway defines its pathogenesis; analysis of the gene expression profiles were associated with the G4 $\alpha$ and G4 $\beta$ subcategories. TOX high mobility group box family member 3 , synuclein $\alpha$ interacting protein and, potassium voltage-gated channel interacting protein 4 were identified as three novel potential markers for
\end{abstract}

Correspondence to: Dr Sergio Juárez-Méndez, Laboratory of Experimental Oncology, National Institute of Pediatrics, SSA Research Tower, 3700-C Insurgentes Sur Avenue, Insurgentes Cuicuilco, Mexico City 04530, Mexico

E-mail: ser.mend@gmail.com

Key words: medulloblastoma, microarray, group 4, biomarker, bioinformatics distinguishing the $\alpha$ and $\beta$ subcategories of G4. These genes may be associated with medulloblastoma pathogenesis, and thus may provide a basis for researching novel targeted treatment strategies for G4 medulloblastoma.

\section{Introduction}

Tumors of the central nervous system (CNS) are the most frequent type of solid tumor identified in children. Among them, medulloblastoma has the highest incidence rate, at 6 per 1,000,000 in children aged 1-9 years in the United States (1), which is 10 -fold greater than the incidence rate in adults (2). Since the organogenesis of the cerebellum is strongly associated with its susceptibility to oncogenic transformation, medulloblastomas are classified as embryonic tumors, which is consistent with the high incidence rate of tumors derived from this organ in children $(3,4)$. Conventionally, the histological classification of medulloblastoma has been established to comprise the following variants: Classic, desmoplastic/nodular, anaplastic, large-cell and medulloblastoma with extensive nodularity (5). Current treatment options include surgery, radiotherapy and chemotherapy, and treatment is selected based on patient age, among other risk factors (6). These strategies typically achieve a 5-year survival rate of 50-80\%; however, severe neurological secondary effects can result from such treatments (6-8).

The discovery of the molecular landscape of medulloblastoma has provided a new perspective in the research of the disease. This has allowed the elucidation of the origin of medulloblastoma, and how specific molecular dysregulation can affect the treatment response and subsequent clinical outcomes. The molecular classification of medulloblastoma is based on 4 subgroups: WNT, SHH, Group 3 (G3) and Group 4 (G4). The WNT subgroup is associated with the dysregulation of Wnt signaling; it accounts for $10 \%$ of all medulloblastoma cases and is associated with the most positive prognosis $(9,10)$. This subgroup is characterized by mutations in catenin $\beta 1, A P C$ and Frizzled, among other genes, which are known to maintain and activate pathways involving the $\beta$-catenin protein, a transcription factor upregulating cell proliferation, survival and migration (11). Additionally, the WNT subgroup is associated with a predisposition to the 
development of Turcot syndrome, and may also be associated with deletions in chromosome 6 (12-14). The SHH subgroup is characterized by the dysregulation of the sonic hedgehog pathway. It accounts for $30 \%$ of all medulloblastomas, and is associated with an intermediate prognosis $(9,10)$. SHH medulloblastomas are also associated with Gorlin syndrome $(15,16)$. SHH medulloblastomas principally exhibit the inactivation of patched 1 and suppressor of fused homolog, missense mutations in smoothened (SMO), and amplifications of GLI family zinc finger 2. These genetic alterations promote the translocation of the transcription factor, GLI, into the nucleus, which can lead to the activation of cancer driver genes (17). WNT and $\mathrm{SHH}$ are the best characterized subgroups of medulloblastoma, which may enable specific therapeutic targeting.

Group 3 (G3) represents $\sim 25 \%$ of medulloblastoma cases, and is characterized by amplifications in $M Y C N$ and orthodenticle homeobox 2 (OTX2), the gain of chromosomes 7 and 17q, and the loss of chromosomes $10 \mathrm{q}$ or $16 \mathrm{q}$ (9). Group 4 (G4) is the most common subgroup, accounting for $\sim 35 \%$ of all cases $(9,10)$; it is biologically heterogeneous and difficult to characterize due to its molecular markers being shared with G3 and SHH. These markers include mutations in lysine demethylase, and amplifications of OTX2 or lysine methyltransferase 2D, among others. G3 and G4 are associated with a relatively poor prognosis compared with the WNT and SHH subgroups (9).

The high heterogeneity and prevalence of G3 and G4 medulloblastomas highlight the requirement to establish molecular classifications for each subgroup based on specific markers detectable by inexpensive techniques. Ellison et al (18) reported an immunohistochemical method for distinguishing between SHH/WNT and non-SHH/WNT tumors. This method considers specific markers of each subgroup, including GRB2 associated binding protein $1, \beta$-catenin, filamin A and Yes associated protein 1 . However, it cannot differentiate between the G3 and G4 subgroups. The identification of novel biomarkers would increase the efficiency of molecular diagnosis, and may be achieved through the identification and characterization of the key pathways that are distinctly dysregulated in the G3 or G4 subgroups.

In the present study, an in silico analysis of the 4 molecular subgroups of medulloblastoma was performed with the aim of characterizing potential novel markers for the identification of each subgroup, with an emphasis on the characterization of G4. A number of key markers were identified, allowing the classification of further subdivisions within G4. This data may contribute to the more effective classification of medulloblastoma, and aid in the identification of alternative therapeutic targets, particularly for G4 medulloblastoma.

\section{Materials and methods}

Datasets. Affymetrix microarray U133_Plus 2 Array data was downloaded from the Gene Expression Omnibus database (http://www.ncbi.nlm.nih.gov/geo). This included medulloblastoma and healthy control cerebellar tissue (CT) data. The microarray data included various characteristics, including the sample information (tissue type and disease status), RNA purification method, RNA quality, RNA integrity, RNA concentration and the microarray protocol used. Tumor expression profiles were grouped according to the medulloblastoma molecular subgroups. The following datasets were included in the present study: GSE4036, GSE10327 (19-21), GSE37418 (20-22), GSE44971 (23) and GSE49243 (20,24).

Data analysis. Bioinformatics analysis was performed using the Partek Genomics Suite version 6.6 (Partek, Inc., St Louis, Missouri, USA). In brief, all datasets were uploaded to the Partek Genomics Suite and summarization was performed with Median Polish analysis with quantile normalization and background correction using a robust multiarray average. The probe set was then transformed to $\log _{2}$. A second selection of the microarray dataset was performed based on an analysis by Quality Control Microarray processing according to a previous study (25). In the aforementioned analysis the fluorescence intensity of the microarray quality control was evaluated using bioB, bioC, bioD and Cre, at final concentrations of 1.5, 5, 25 and $100 \mathrm{pM}$, respectively. Additionally, Poly-A RNA controls were evaluated using Dap, Thr, Phe and Lys to final concentrations of 1:7,500, 1:25,000, 1:50,000 and 1:100,000, respectively. Microarrays without the relative signal intensities of Poly-A RNA and hybridization controls were excluded from the present study.

Differentially expressed genes were determined using the data from healthy CT to define baseline expression. The geometric least squares means model was used for the following independent analyses: WNT vs. CT; SHH vs. CT; G3 vs. CT; and G4 vs. CT. Differential gene expression was also detected between G3 and G4 using G3 as the baseline, between the subsequently defined subgroups G4 $\alpha$ and G4 $\beta$ using G4 $\beta$ as the baseline, and between WNT, SHH, G3, G4 $\alpha$ and G4 $\beta$, using CT as the baseline. Significantly differentially expressed genes were detected by an analysis of variance using a false discovery rate threshold of $<0.005$ and fold change (FC) thresholds of $<-5$ and $>5$. Hierarchical clustering heatmaps were constructed using the Euclidean distance method.

Systems biology. The differentially expressed genes were uploaded to the MetaCore portal (Thomson Reuters, New York City, NY, USA) for enrichment analysis, in which FC was used as the parameter for differential expression. Significant signaling pathways were selected using a Z-score, as outlined in our previous study (26).

\section{Results}

Medulloblastoma expression profiles can be grouped based on the molecular subgroups. Initially, the data from 237 microarray expression profiles of medulloblastoma tumor and CT samples were downloaded. All microarrays were subjected to quality control analysis; $~ 42 \%$ of all arrays were excluded based on the Poly-A RNA controls. The signal intensity demonstrated a high level of variation, as previously reported $(25,27)$.

A total of 137 expression profiles were included in the analysis, including profiles from the following molecular subgroups: WNT, $n=17$; SHH, n=27; G3, n=20; G4, n=53 and healthy $\mathrm{CT}, \mathrm{n}=20$ (Table I). The medulloblastoma samples were successfully grouped based on their molecular classification using the CT group as a reference control (Fig. 1). 
Table I. DEGs in molecular subgroups compared with the control cerebellar tissues $(n=20)$.

\begin{tabular}{lcccc}
\hline & & \multicolumn{3}{c}{ DEGs, $\mathrm{n}$} \\
$\begin{array}{l}\text { Subgroup } \\
\text { name }\end{array}$ & $\begin{array}{c}\text { Samples, } \\
\mathrm{n}\end{array}$ & Upregulated & Downregulated & Total \\
\hline WNT & 17 & 1,073 & 1,092 & 2,165 \\
SHH & 27 & 728 & 991 & 1,719 \\
G3 & 20 & 673 & 1,117 & 1,790 \\
G4 & 53 & 602 & 801 & 1,403 \\
\hline
\end{tabular}

DEG, differentially expressed genes; G3, group 3 medulloblastoma; G4, group 4 medulloblastoma.

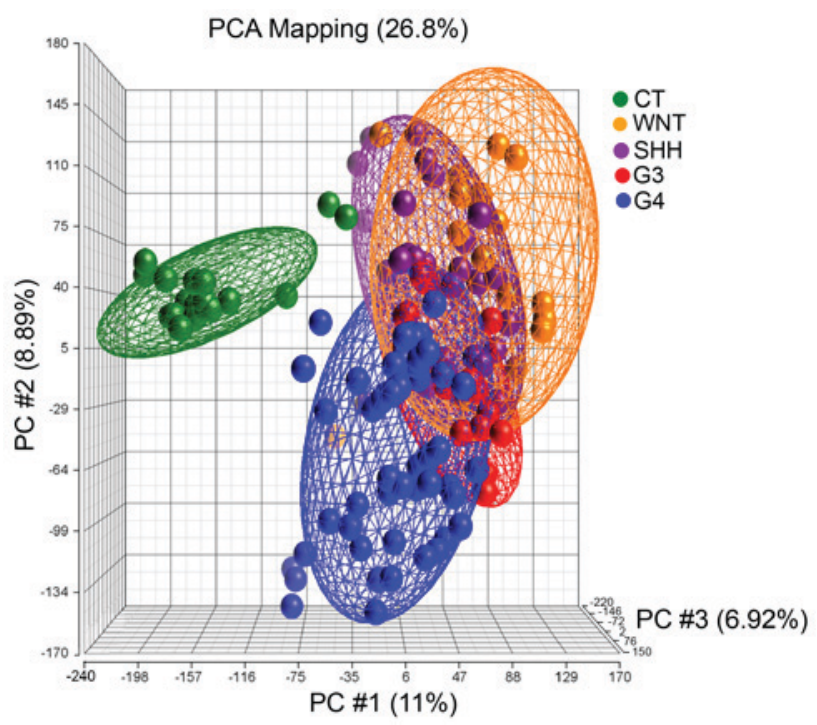

Figure 1. Gene expression patterns in medulloblastoma subgroups. PCA was performed with an expectation-maximization algorithm in Partek Genomics Suite v6.6. The PCA depicts the overlapping of gene expression between the 4 molecular subgroups: WNT, orange; SHH, purple; G3, red; G4, blue; and $\mathrm{CT}$, green. PCA, principal component analysis; $\mathrm{CT}$, control cerebellar tissue.

Gene expression profiling revealed potential targets for molecular subgroup classification. The expression profiles of the medulloblastoma molecular subgroups compared with healthy CT revealed numerous differences. The number of upregulated or downregulated differentially expressed genes were as follows: WNT, $n=2,165 ; \mathrm{SHH}, \mathrm{n}=1,719 ; \mathrm{G} 3, \mathrm{n}=1,790$; and G4, n=1,403 (Fig. 2; Table I). The transcripts overexpressed in the WNT subgroup included frizzled class receptor 10 (FC, 55.2), Dickkopf WNT signaling pathway inhibitor 1 (FC, 16.6), forkhead box D1 (FC, 10.6), ALK receptor tyrosine kinase (FC, 6.4) and forkhead box L2 (FC, 6.3; Fig. 3A). Claudin 1 (FC, 12.33), ADAM metallopeptidase with thrombospondin type 1 motif 6 (FC, 11.43) and growth arrest specific 1 (FC, 8.19) were upregulated in the SHH subgroup (Fig. 3B); FEZ family zinc finger 2 (FC, 6.83), ADP Ribosylation Factor Like GTPase 4D (FC, 6.41) and sperm associated antigen 5 (FC, 5.25) (Fig. 3C) were upregulated in G3; G4 exhibited the overexpression of caspase recruitment domain family member 10 (FC, 10.23), CXXC finger protein 4 (FC, 6.64), SH2 domain containing adaptor protein $\mathrm{B}(\mathrm{FC}, 5.92)$ and $\mathrm{CXC}$ motif chemokine ligand 14 (FC, 5.38; Fig. 3D).

Differential gene expression between $G 3$ and $G 4$ reveals a novel subgroup in G4. The molecular classification of medulloblastoma and other types of CNS tumor was previously investigated (28). Defining the expression profiles associated with G3 and G4 has been a particular challenge in medulloblastoma research, as the groups share several molecular characteristics and are considered the most internally heterogeneous (29). In order to identify potential molecular markers associated with G3 and G4, G3 was used for a comparative analysis against $\mathrm{G} 4$. The analysis identified 224 differentially expressed genes; 142 were upregulated and 82 were downregulated in G4 compared with G3 (Fig. 4). The differentially expressed genes allowed clustering into 2 distinct groups, representing G3 and G4, as illustrated by the heatmap. However, the G4 cluster unexpectedly demonstrated 2 profiles, with a small secondary cluster representing 12 samples ( 25\%; Fig. 4).

The results indicated a potential sub-classification of G4. In order to identify the molecular differences within G4, G4 samples were sub-classified as G4 $\alpha$ and G4 $\beta$ [36 (75\%) and 12 samples (25\%), respectively; (Fig. 5)]. A comparative analysis was performed using G4 $\beta$ as the baseline against G4 $\alpha$. The analysis identified a differential expression profile for $\mathrm{G} 4 \alpha$, with 3 genes upregulated and 33 downregulated (Table II). The 5 molecular subgroups of medulloblastoma were integrated for a comparative analysis using the $\mathrm{CT}$ profile as the baseline against WNT, SHH, G3, G4 $\alpha$, and G4 $\beta$. The WNT, SHH, and G3 subgroups retained the same molecular profiles as the first analysis of only 4 subgroups, whereas the G4 $\alpha$ and G4 $\beta$ subgroups demonstrated 1,418 and 1,320 differentially expressed genes, respectively (Fig. 6). However, a number of molecules shared expression patterns among the molecular subgroups, including $\mathrm{G} 4 \alpha$ and $\mathrm{G} 4 \beta$, which would be expected, as molecular similarities between medulloblastoma tumors of all types are inevitable (Table III).

Enrichment analysis demonstrated potential therapeutic targets associated with medulloblastoma subgroups. Enrichment analysis enabled the identification of gene profiles and pathways shared between each molecular subtype of medulloblastoma (Fig. 7). The most common medulloblastoma-associated process was 'cell cycle', associated with the upregulation of kinesin family member 11, cell division cycle 20, mitotic arrest deficient 2 like 1, kinetochore complex component and cyclin B1 (Fig. 7A). The 'renin-angiotensin pathway' was significantly downregulated, which was associated with the downregulated expression of angiotensinogen and angiotensins I, II, III, IV, -(2-10), -(1-7) and -(1-9) (Fig. 7B). However, differences in the level of expression existed between the subgroups, consistent with the subclassifications described in the present study (Fig. 7A and B).

\section{Discussion}

This data mining analysis of microarray gene expression data enabled the identification of a range of dysregulated molecules associated with each subgroup of medulloblastoma, as has 


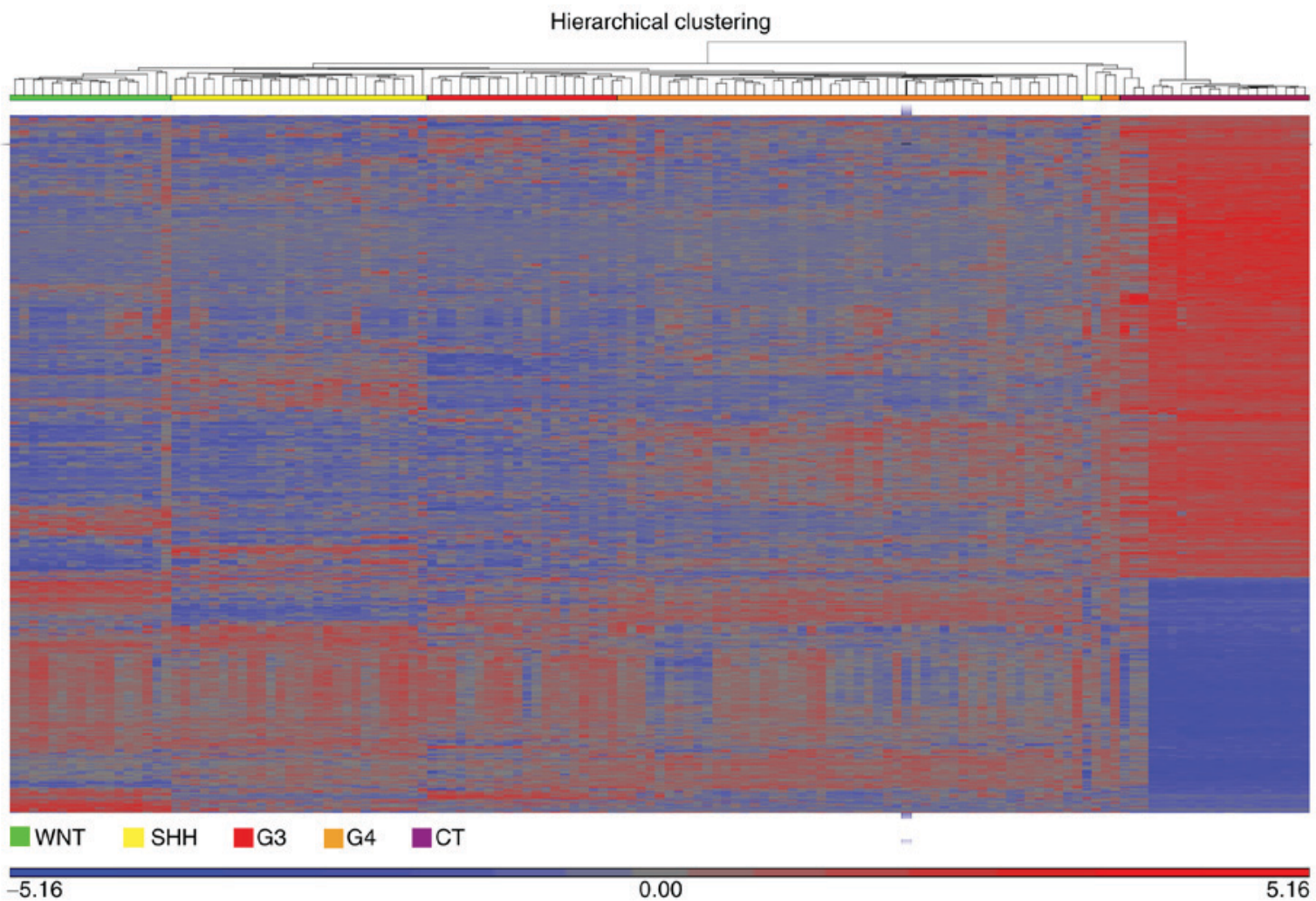

Figure 2. Dysregulated genes in medulloblastoma. The heatmap illustrates the differentially expressed genes in the 4 molecular subgroups of medulloblastoma compared with CT. Differential gene expression was identified by an analysis of variance. The plot was generated for up- and downregulated genes using a fold change threshold of $>5$ or $<-5$, and a false discovery rate threshold of $<0.005$. The profiles of the WNT, SHH, G3 and G4 subgroups were examined against the baseline CT profile using the geometric least squares means model. WNT, green; SHH, yellow; G3, red; G4, orange; and CT, purple. CT, control cerebellar tissue; G3, group 3 medulloblastoma; G4, group 4 medulloblastoma.

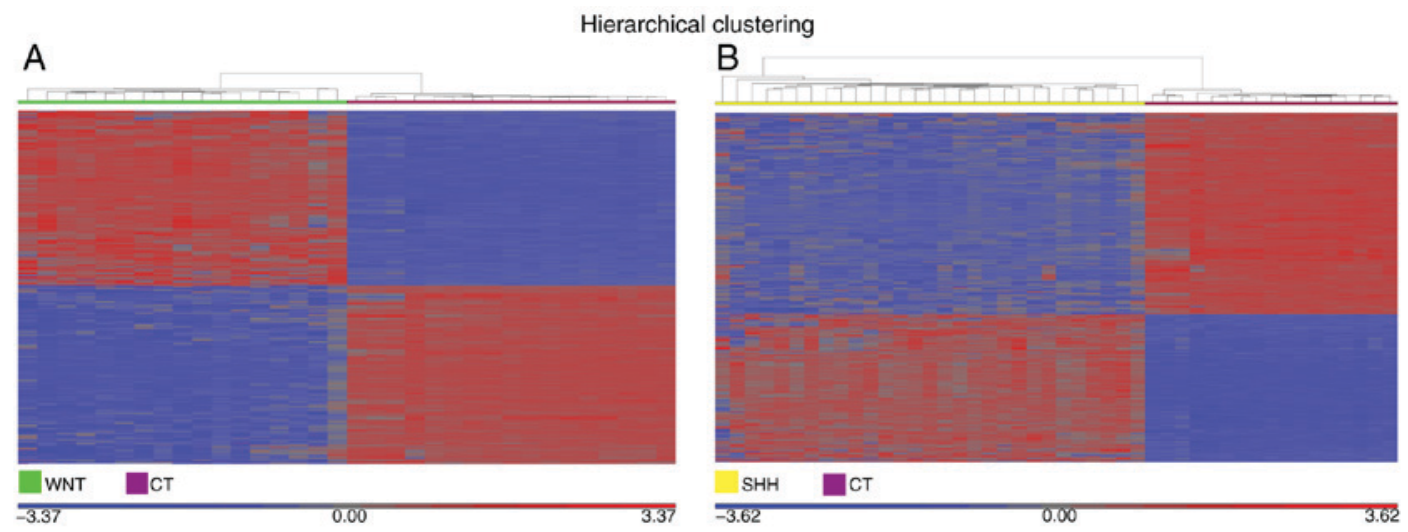

C

D
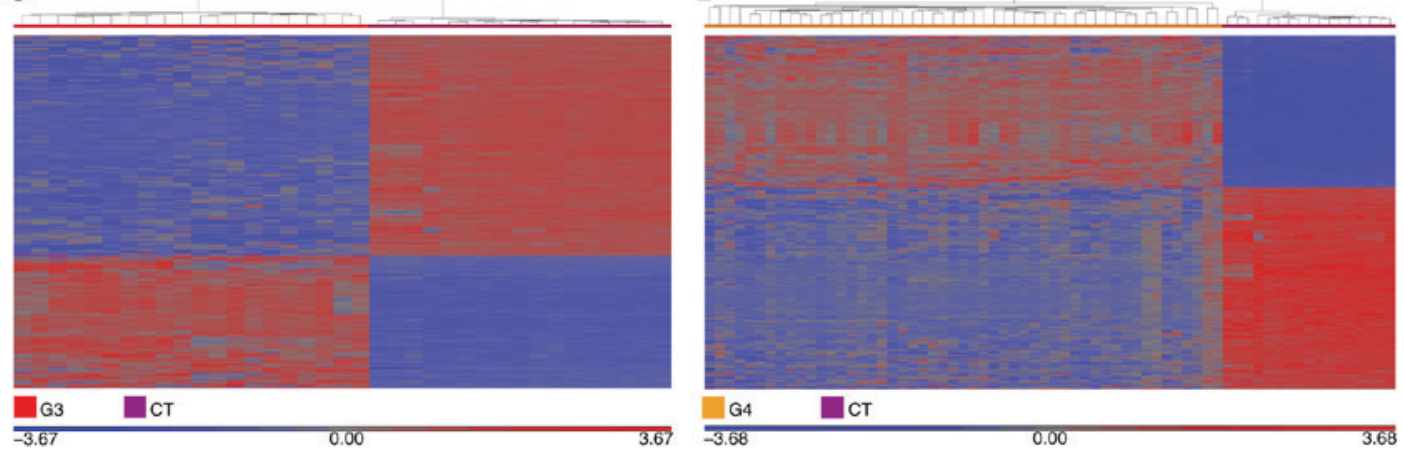

Figure 3. Gene expression profiles associated with the molecular subgroups of medulloblastoma. The heatmaps illustrate the differentially expressed genes for the (A) WNT, (B) SHH, (C) G3 and (D) G4 subgroups, each compared with CT. The heat maps were constructed using the geometric least squares means model. Significant differential expression values were included only when the fold change was $>5$ or $<-5$ and the false discovery rate was $<0.005$. CT, control cerebellar tissue; G3, group 3 medulloblastoma; G4, group 4 medulloblastoma. 
Hierarchical clustering

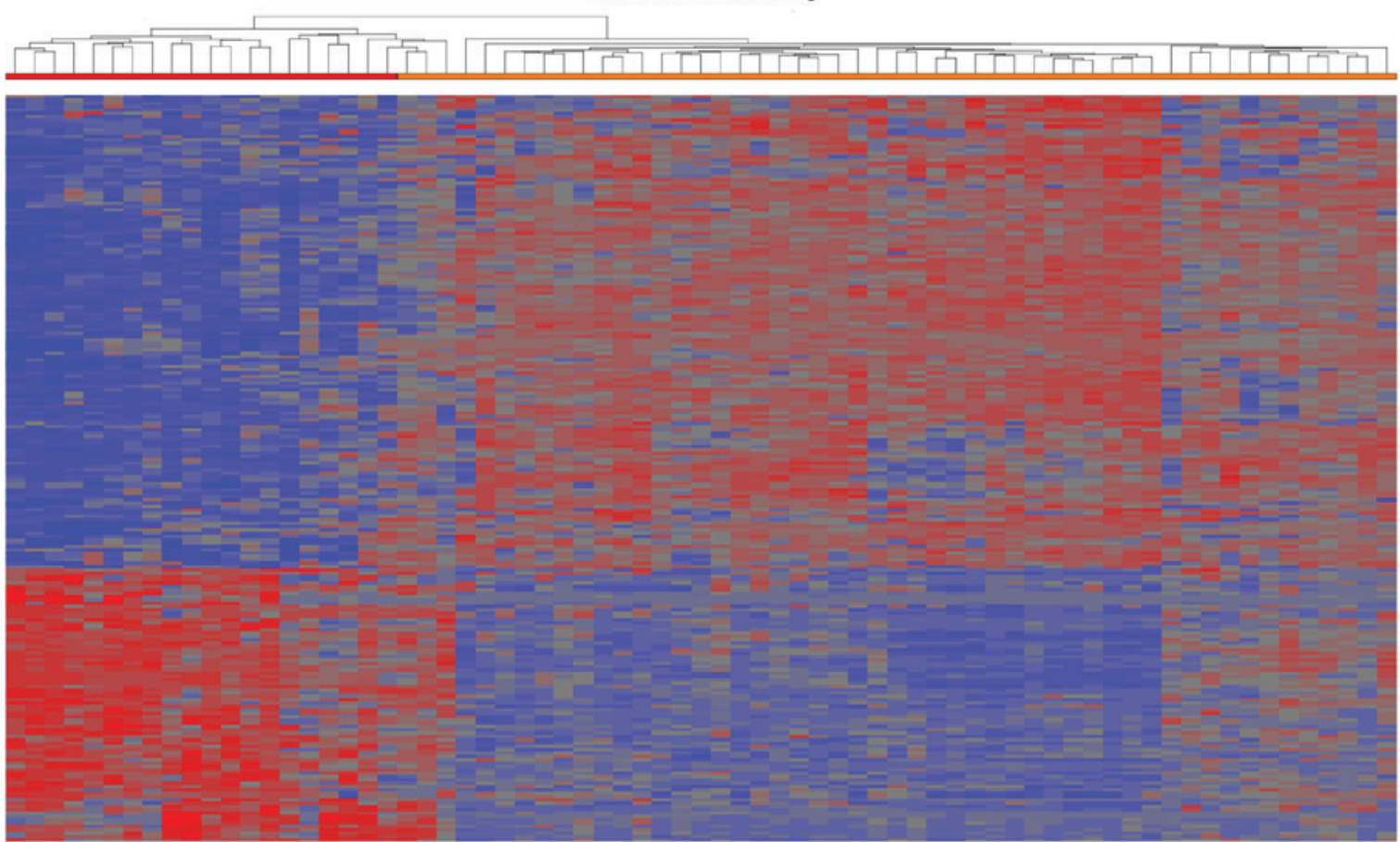

Figure 4. Hierarchical clustering of the molecular subgroups G3 and G4. The heatmap depicts the differentially expressed genes in G3 compared with G4, as evaluated by analysis of variance using the geometric least squares means model, with G3 as the baseline. Differential expression was indicated when the fold change was $>5$ or $<-5$ and the false discovery rate was $<0.005$. Clustering analysis identified two subgroups within G4 based on the dissimilarity of samples as assessed using a Euclidean model by means of average linkage, which represented 48 tumors in total. A total of $12 \mathrm{G} 4$ samples exhibited significantly different gene expression profiles compared with the remaining 36. G3, group 3 medulloblastoma; G4, group 4 medulloblastoma.

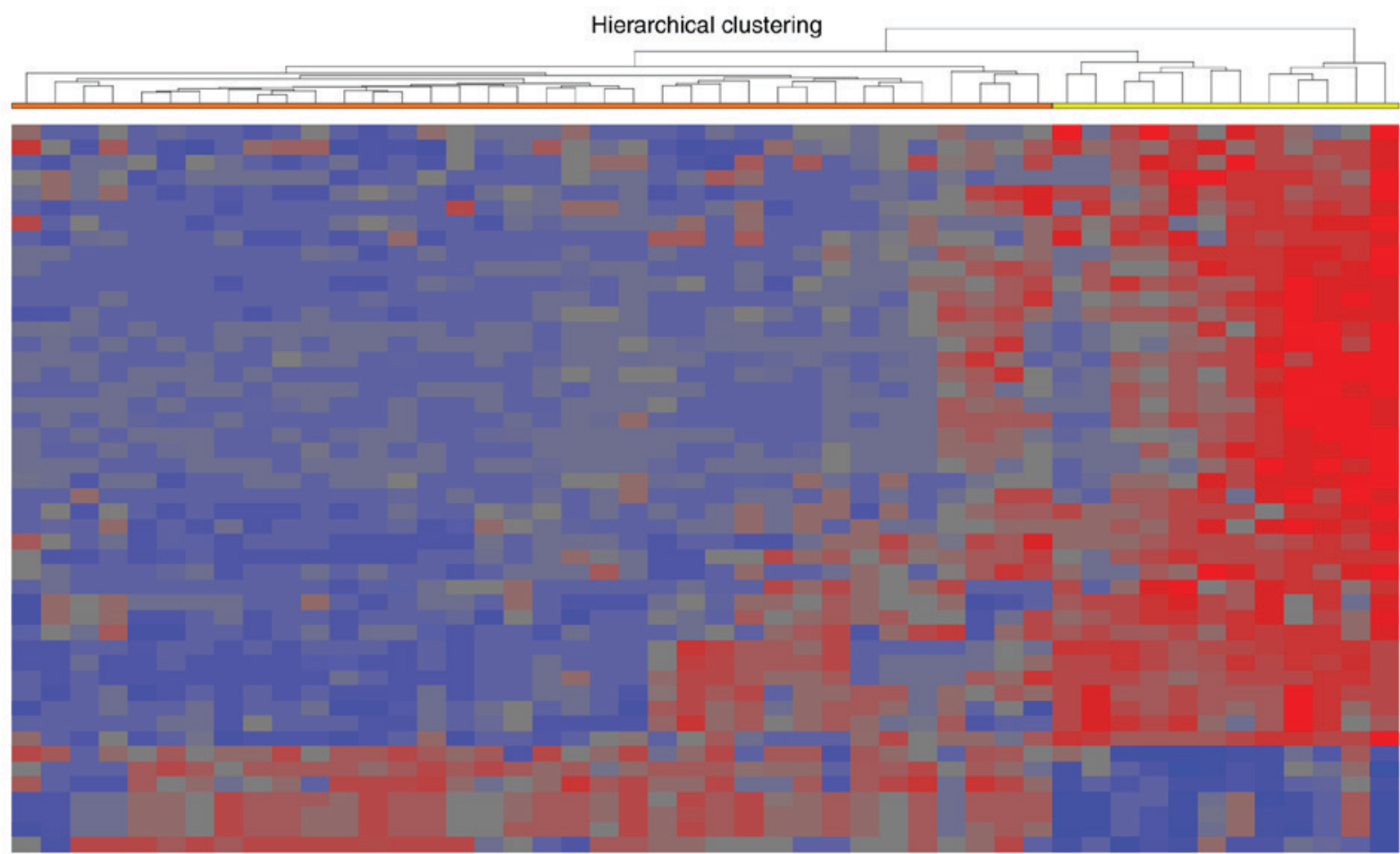

\section{$\square \mathrm{G} 4 \alpha \quad \mathrm{G} 4 \beta$}

Figure 5. Fractionation of G4 medulloblastoma. The heatmap illustrates 48 dysregulated genes in G4 medulloblastoma. The dissimilarity of samples based on average linkage indicated the fractionation of G4 into the potential subgroupings of G4 $\alpha$ (left; orange bar) and G4 $\beta$ (right; yellow bar). Differential expression values were included when the fold change was $>5$ or $<-5$ and the false discovery rate was $<0.005$. G3, group 3 medulloblastoma; G4, group 4 medulloblastoma. 
Table II. Differentially expressed genes in the G4 $\alpha$ subgroup compared with the G4 $\beta$ subgroup.

Gene symbol

Gene name

Fold change

TOX3

TOX high mobility group box family member 3

(G4 $\alpha$ vs. G4 $\beta$ )

SNCAIP

KCNIP4

PLXDC1

BCL11B

ABCA4

GALNT10

GLCCI1

FAM126A

SLC1A7

GALNT14

IGSF11

SHROOM2

CNTN3

GNGT2

AIPL1

RASGRF2

RP1

NR2E3

CRX

LAPTM4B

PTPN13

PDC

RCVRN

ANO2

MPP4

RD3

FAM19A4

CDHR 1

CABP5

ZNF385B

IMPG2

PRSS12

SST

NRL PDE6H
Synuclein $\alpha$ interacting protein

8.61506

Potassium voltage-gated channel interacting protein 4

6.85187

Plexin domain containing 1

B-cell CLL/lymphoma 11B

6.27879

$-3.02931$

ATP-binding cassette sub-family A member 4

$-3.06849$

$-3.07119$

Polypeptide N-acetylgalactosaminyltransferase 10

$-3.13204$

Glucocorticoid induced transcript 1

$-3.22556$

Family with sequence similarity 126 , member A

$-3.24904$

Solute carrier family 1 , member 7

$-3.29459$

Polypeptide N-acetylgalactosaminyltransferase 14

$-3.33326$

Immunoglobulin superfamily, member 11

$-3.33354$

Shroom family member 2

$-3.39392$

Contactin 3

$-3.83419$

G protein subunit $\gamma$ transducin 2

$-3.85892$

$-3.99598$

$-4.01560$

$-4.04201$

$-4.36478$

$-4.41841$

$-4.56260$

$-4.76854$

$-4.80708$

$-4.91791$

$-5.11475$

$-5.39617$

$-5.39937$

$-5.76661$

Family with sequence similarity 19, member A4,

C-C motif chemokine-like

Cadherin-related family member 1

$-5.80075$

Calcium binding protein 5

$-7.79064$

$-8.85360$

$-9.61426$

$-9.84918$

$-9.92109$

$-10.53520$

$-11.56650$

G4, group 4 medulloblastoma.

been previously achieved $(9,28)$. Furthermore, a potential subcategorization of G4 was defined. G4 medulloblastoma is the most frequently occurring molecular subgroup, as well as one of the most heterogeneous, as no definitive pathway defines its pathogenesis.

Despite the classification of medulloblastoma into 4 subgroups, the heterogeneity of G3 and G4 and their association with poorer prognoses remain unaddressed. Thompson et al (30) described 5 subgroups instead of the standard 4, suggesting some overlap between the G3 and G4 subgroups and/or a subdivision of G4 itself. Additionally, Cho et al (31) reported an analysis of the mRNA transcriptome via a high-density single-nucleotide polymorphism array and miRNA analysis, in which 6 molecular subgroups were identified; the WNT and SHH subgroups remained the same, whereas $\mathrm{G} 3$ was subclassified into the $\mathrm{C} 1 / \mathrm{C} 5$ subgroups and G4 into the $\mathrm{C} 2 / \mathrm{C} 4$ subgroups, reflecting the lack of homogeneity in the G3 and G4 subgroups. These C2 and C4 G4 subcategories may be equivalent to those described in the present study. 
Table III. Potential therapeutic targets in medulloblastoma tumors, including the top ten upregulated and downregulated differentially expressed genes in the 5 subgroups of medulloblastoma.

Fold change vs. control cerebellar tissue

\begin{tabular}{|c|c|c|c|c|c|}
\hline \multirow[b]{2}{*}{ Gene symbol } & \\
\hline & WNT & SHH & G3 & $\mathrm{G} 4 \alpha$ & G4 $\beta$ \\
\hline SOX11 & 50.4508 & 159.2550 & 114.7920 & 214.6350 & 157.7430 \\
\hline LHX2 & 19.0600 & 18.1300 & 62.1164 & 118.7020 & 45.9915 \\
\hline KIAA0101 & 89.8103 & 97.5187 & 119.3500 & 106.7370 & 70.2860 \\
\hline TOP2A & 97.4276 & 74.4959 & 95.3006 & 89.4034 & 56.8238 \\
\hline TMSB15A/B & 27.3665 & 131.5370 & 36.4977 & 80.2035 & 74.1441 \\
\hline DACH1 & 25.9512 & 23.0074 & 28.4259 & 74.5587 & 41.3941 \\
\hline SOX4 & 13.2496 & 15.9908 & 25.3652 & 64.0049 & 53.1116 \\
\hline DACH1 & 19.2667 & 14.5598 & 16.1719 & 61.2870 & 21.7303 \\
\hline RRM2 & 48.8038 & 47.4022 & 75.8280 & 55.5282 & 46.5205 \\
\hline IGFBPL1 & 68.8801 & 60.7771 & 10.3575 & 50.0114 & 48.5435 \\
\hline ITPR1 & -26.0343 & -27.0244 & -78.6472 & -58.7188 & -47.0085 \\
\hline SYT1 & -8.2123 & -8.1780 & -25.8050 & -62.9443 & -37.5653 \\
\hline SPHKAP & -98.0768 & -21.4717 & -91.2947 & -64.4953 & -42.0679 \\
\hline PDE1A & -119.2340 & -13.2837 & -128.5230 & -66.5903 & -25.8941 \\
\hline CDR1 & -78.4458 & -66.2995 & -75.0443 & -83.1560 & -78.4922 \\
\hline CBLN3 & -82.5110 & -97.7424 & -131.9180 & -97.2893 & -71.7417 \\
\hline CRTAM & -142.5620 & -119.6880 & -176.9190 & -98.6054 & -91.3960 \\
\hline CALB1 & -90.1248 & -100.8540 & -50.7010 & -121.3370 & -113.9070 \\
\hline PVALB & -125.1260 & -90.7036 & -243.5880 & -175.7630 & -113.7100 \\
\hline GABRA6 & -254.6810 & -245.7720 & -171.3970 & -192.7920 & -133.8460 \\
\hline
\end{tabular}

G3, group 3 medulloblastoma; G4, group 4 medulloblastoma.

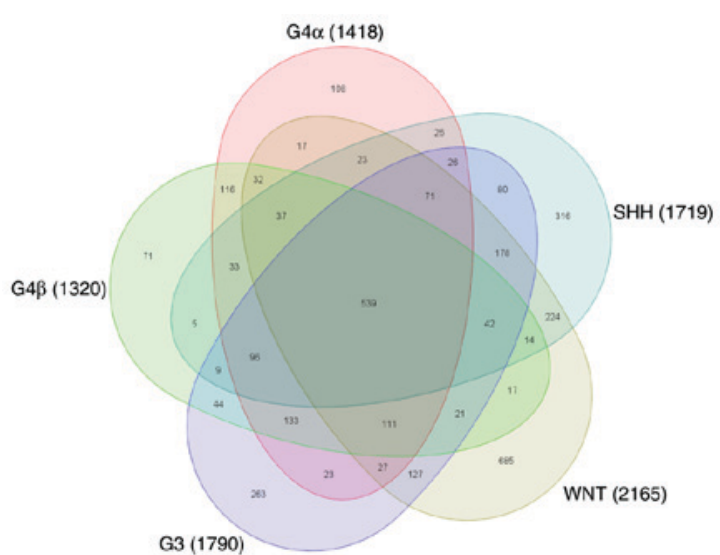

Figure 6. Gene expression profiles associated with the molecular subgroups of medulloblastoma. The Venn diagram represents the 5 subgroups of medulloblastoma. A total of 539 dysregulated genes were common between the 5 subgroups. Distinct differentially expressed genes was identified for WNT $(n=685)$, SHH $(n=316)$, G3 $(n=263), G 4 \alpha(n=108)$ and G4 $\beta(n=71)$. G3, group 3 medulloblastoma; G4, group 4 medulloblastoma.

It has been indicated that G3 and G4 medulloblastoma express gene members of pathways associated with photoreception and neuronal function, thus suggesting a grade of differentiation (32). In the present study, the overexpression of TOX high mobility group box family member 3 (TOX3), potassium voltage-gated channel interacting protein 4 (KCNIP4), and synuclein $\alpha$ interacting protein $(S N C A I P)$ was identified in the G $4 \alpha$ group compared with G4 $\beta$. TOX3 is important in the development of the CNS (33) and its expression is correlated with neuronal markers, including Nestin and SOX2. The TOX3 protein participates in the regulation of DNA structure and coiling, and thus aids in transcriptional regulation. For instance, TOX3 interacts with the CAMP Responsive Element Binding Protein (CREB)/CREB binding protein complex and regulates the transcription of genes dependent on calcium ions, and is associated with neuronal survival (34). In addition, TOX3 is expressed in breast and lung cancer (35).

The overexpression of KCNIP4 was identified in the G4 $\alpha$ subgroup only. The KCNIP family comprises a group of small calcium-binding proteins. KCNIP4 encodes a potassium ion transporter directly associated with neuronal function, which interacts with presenilin, a protein associated with early-onset familial Alzheimer's disease (36). To date, KCNAl (10) and KCNJ2 (37) have been reported as potential markers of G4 medulloblastoma; however, to the best of our knowledge, this is the first time that KCNIP4 has been identified as specifically associated with the $\mathrm{G} 4 \alpha$ subgroup of medulloblastoma.

SNCAIP has been described by Northcott et al (37) as a key gene in the subcategorization of G4 medulloblastoma, which is supported by the results of the present study. Northcott et al (37), reported that a tandem duplication of SNCAIP defined a novel subtype of G4 medulloblastoma, $4 \alpha$, which comprised $10.4 \%$ of G4 medulloblastoma cases among 

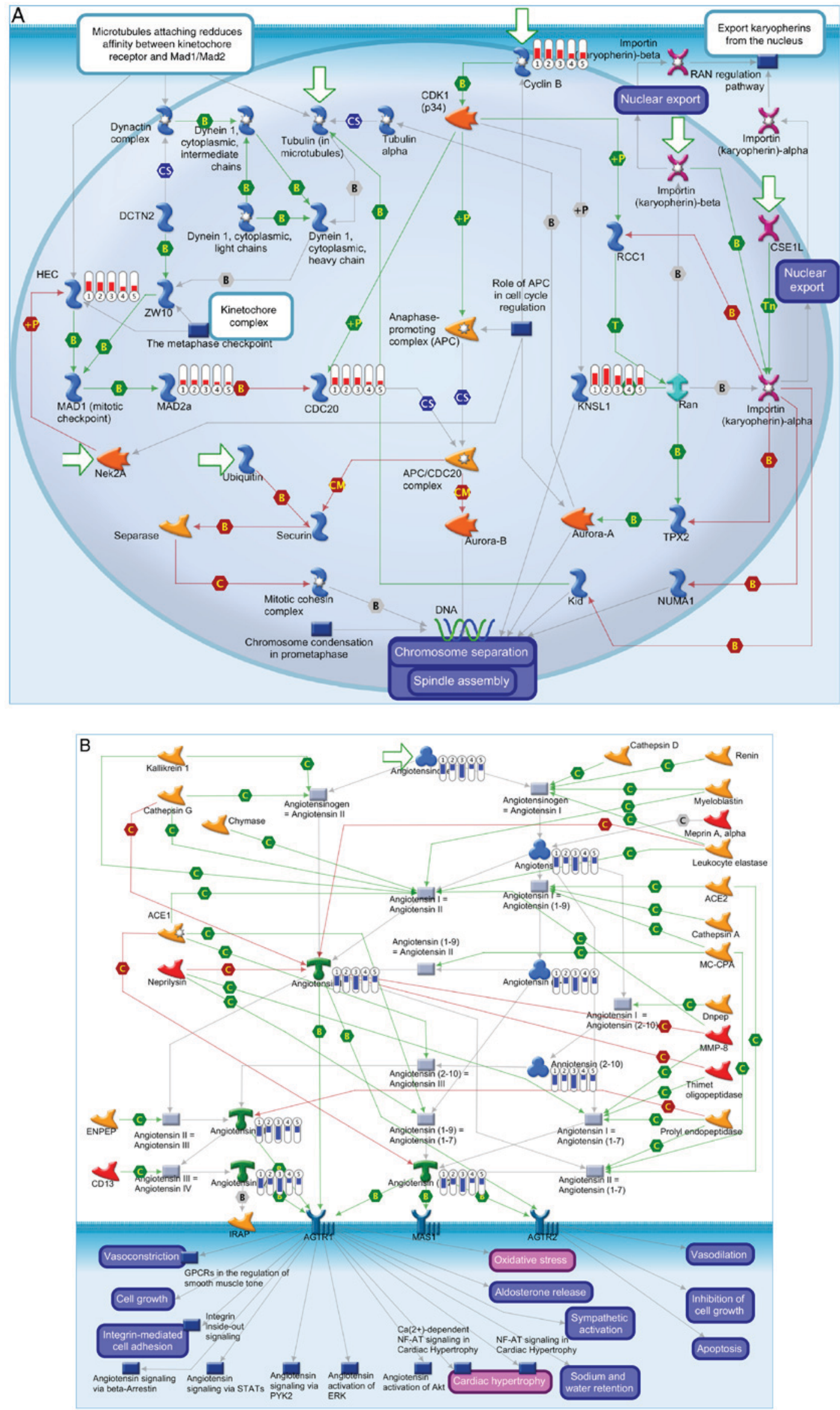

Figure 7. Enrichment analysis of the differentially expressed genes in the 5 subgroups of medulloblastoma. The thermometers indicate expression (red, upregulated; blue, downregulated). The numbers in the thermometers indicate the molecular subgroups (1, WNT; 2 , SHH; 3 , group $3 ; 4$, group $4 \alpha$ and 5 , group $4 \beta$ medulloblastoma). (A) Canonical pathways of the upregulated differentially expressed genes in the 5 molecular subgroups of medulloblastoma. (B) Canonical pathways of the downregulated differentially expressed genes in the 5 molecular subgroups of medulloblastoma. 
1,087 medulloblastoma genomes. The SNCAIP duplication was associated with a 1.5 -fold increase in SNCAIP expression in G $4 \alpha$ compared with G4 $\beta$. Significantly, SNCAIP duplications are mutually exclusive with $M Y C N$ and $C D K 6$ amplifications (37). It has been established that synphilin-1 in neurons interacts with $\alpha$-synuclein, a protein present in Lewy bodies in patients with Parkinson's disease (38).

Northcott et al (37) also reported that somatic copy number aberrations (SCNAs) were common in medulloblastoma and were enriched depending on the subgroup. Specific SCNAs were associated with TGF- $\beta$ in G3, and NF-kB in G4, which may enable the identification of novel targets for the specific treatment of different subgroups of medulloblastoma. Despite the existing specific therapies for $\mathrm{SHH}$ medulloblastoma, including GDC-0449, an inhibitor of the SHH pathway that targets the SMO receptor (39), it is necessary to identify alternative therapies for all the molecular subgroups, particularly for those associated with a relatively poor prognosis, including G3 and G4. The markers identified in the present study, together with other markers reported elsewhere, may form the basis for new approaches to treat medulloblastoma.

Further studies are required to verify the potential of the markers identified in the present study to define G4 subcategories. It will be important to validate the presence of these markers in patient samples, and explore whether they are associated with a relatively poor prognosis in medulloblastoma, as well as how they participate in the physiopathology of the disease.

In conclusion, three novel potential markers to differentiate G4 medulloblastoma into two subclassifications, TOX3, KCNIP4 and SNCAIP, have been identified in the present study. This may provide a basis for future studies on the characterization of G4 medulloblastomas. Future research should focus on these genes as potential biomarkers, in the pathogenesis of medulloblastoma, and as targets for the development of targeted therapies.

\section{Acknowledgements}

Not applicable.

\section{Funding}

The present study was supported by CONACyT (grant no., SALUD-2014-1-233868) and by federal funds of the National Institute of Pediatrics (protocol no. 036/2014 and 37/2015). Rosa Angélica Castillo-Rodríguez was supported by Cátedras-CONACyT (project no. 1059).

\section{Availability of data and materials}

The datasets analyzed during the current study are public and available in the Gene Expression Omnibus database, www. ncbi.nlm.nih.gov/geo (accession nos. GSE4036, GSE10327, GSE37418, GSE44971 and GSE49243).

\section{Authors' contributions}

RACR and SJM conceived and designed the work. RACR, SJM, and VMDB acquired, analyzed, and interpreted the data.
All authors discussed and wrote the manuscript. All authors read and approved the final manuscript.

\section{Ethics approval and consent to participate}

Not applicable.

\section{Consent for publication}

Not applicable.

\section{Competing interests}

The authors declare that they have no competing interests.

\section{References}

1. Smoll NR: Relative survival of childhood and adult medulloblastomas and primitive neuroectodermal tumors (PNETs). Cancer 118: 1313-1322, 2012.

2. Smoll NR and Drummond KJ: The incidence of medulloblastomas and primitive neurectodermal tumours in adults and children. J Clin Neurosci 19: 1541-1544, 2012.

3. Louis DN, Perry A, Reifenberger G, von Deimling A, Figarella-Branger D, Cavenee WK, Ohgaki H, Wiestler OD, Kleihues P and Ellison DW: The 2016 world health organization classification of tumors of the central nervous system: A summary. Acta Neuropathol 131: 803-820, 2016.

4. Scotting PJ, Walker DA and Perilongo G: Childhood solid tumours: A developmental disorder. Nat Rev Cancer 5: 481-488, 2005.

5. Gilbertson RJ and Ellison DW: The origins of medulloblastoma subtypes. Annu Rev Pathol 3: 341-365, 2008.

6. Childhood Central Nervous System Embryonal Tumors Treatment (PDQ $\left.{ }^{\circledR}\right)$ : Health Professional Version: PDQ pediatric treatment editorial board. In: PDQ Cancer Information Summaries[Internet]. Bethesda (MD): National Cancer Institute (US), 2002.

7. Crawford JR, MacDonald TJ and Packer RJ: Medulloblastoma in childhood: New biological advances. Lancet Neurol 6: 1073-1085, 2007.

8. Paulino AC, Lobo M, Teh BS, Okcu MF, South M, Butler EB, Su J and Chintagumpala M: Ototoxicity after intensity-modulated radiation therapy and cisplatin-based chemotherapy in children with medulloblastoma. Int J Radiat Oncol Biol Phys 78: 1445-1450, 2010.

9. Kool M, Korshunov A, Remke M, Jones DT, Schlanstein M, Northcott PA, Cho YJ, Koster J, Schouten-van Meeteren A, van Vuurden D, et al: Molecular subgroups of medulloblastoma: An international meta-analysis of transcriptome, genetic aberrations, and clinical data of WNT, SHH, Group 3, and Group 4 medulloblastomas. Acta Neuropathol 123: 473-484, 2012.

10. Kijima N and Kanemura Y: Molecular classification of medulloblastoma. Neurol Med Chir (Tokyo) 56: 687-697, 2016.

11. Skowron P, Ramaswamy V and Taylor MD: Genetic and molecular alterations across medulloblastoma subgroups. J Mol Med (Berl) 93: 1075-1084, 2015.

12. Huang H, Mahler-Araujo BM, Sankila A, Chimelli L, Yonekawa Y, Kleihues P and Ohgaki H: APC mutations in sporadic medulloblastomas. Am J Pathol 156: 433-437, 2000.

13. Dahmen RP, Koch A, Denkhaus D, Tonn JC, Sörensen N, Berthold F, Behrens J, Birchmeier W, Wiestler OD and Pietsch T: Deletions of AXIN1, a component of the WNT/wingless pathway, in sporadic medulloblastomas. Cancer Res 61: 7039-7043, 2001.

14. Clifford SC, Lusher ME, Lindsey JC, Langdon JA, Gilbertson RJ, Straughton D and Ellison DW: Wnt/wingless pathway activation and chromosome 6 loss characterize a distinct molecular sub-group of medulloblastomas associated with a favorable prognosis. Cell cycle 5: 2666-2670, 2006.

15. Evans DG, Farndon PA, Burnell LD, Gattamaneni HR and Birch JM: The incidence of gorlin syndrome in 173 consecutive cases of medulloblastoma. Br J Cancer 64: 959-961, 1991.

16. Fujii K and Miyashita T: Gorlin syndrome (nevoid basal cell carcinoma syndrome): Update and literature review. Pediatr Int 56: 667-674, 2014 
17. He X, Zhang L, Chen Y, Remke M, Shih D, Lu F, Wang H, Deng Y, Yu Y, Xia Y, et al: The G protein $\alpha$ subunit Gas is a tumor suppressor in sonic hedgehog-driven medulloblastoma. Nat Med 20: 1035-1042, 2014.

18. Ellison DW, Dalton J, Kocak M, Nicholson SL, Fraga C Neale G, Kenney AM, Brat DJ, Perry A, Yong WH, et al: Medulloblastoma: Clinicopathological correlates of SHH, WNT, and non-SHH/WNT molecular subgroups. Acta Neuropathol 121: 381-396, 2011

19. Kool M, Koster J, Bunt J, Hasselt NE, Lakeman A, van Sluis P, Troost D, Meeteren NS, Caron HN, Cloos J, et al: Integrated genomics identifies five medulloblastoma subtypes with distinct genetic profiles, pathway signatures and clinicopathological features. PLoS One 3: e3088, 2008.

20. Pöschl J, Stark S, Neumann P, Gröbner S, Kawauchi D Jones DT, Northcott PA, Lichter P, Pfister SM, Kool M and Schüller U: Genomic and transcriptomic analyses match medulloblastoma mouse models to their human counterparts. Acta Neuropathol 128: 123-136, 2014.

21. Rack PG, Ni J, Payumo AY, Nguyen V, Crapster JA, Hovestadt V, Kool M, Jones DT, Mich JK, Firestone AJ, et al: Arhgap36-dependent activation of Gli transcription factors. Proc Natl Acad Sci USA 111: 11061-11066, 2014.

22. Robinson G, Parker M, Kranenburg TA, Lu C, Chen X, Ding L, Phoenix TN, Hedlund E, Wei L, Zhu X, et al: Novel mutations target distinct subgroups of medulloblastoma. Nature 488: 43-48, 2012.

23. Lambert SR, Witt H, Hovestadt V, Zucknick M, Kool M, Pearson DM, Korshunov A, Ryzhova M, Ichimura K, Jabado N, et al: Differential expression and methylation of brain developmental genes define location-specific subsets of pilocytic astrocytoma. Acta Neuropathol 126: 291-301, 2013.

24. Kool M, Jones DT, Jäger N, Northcott PA, Pugh TJ, Hovestadt V, Piro RM, Esparza LA, Markant SL, Remke M, et al: Genome sequencing of SHH medulloblastoma predicts genotype-related response to smoothened inhibition. Cancer Cell 25: 393-405, 2014

25. Villegas-Ruiz V, Moreno J, Jacome-Lopez K, Zentella-Dehesa A and Juarez-Mendez S: Quality control usage in high-density microarrays reveals differential gene expression profiles in ovarian cancer. Asian Pac J Cancer Prev 17: 2519-2525, 2016.

26. Villegas-Ruiz V and Juarez-Mendez S: Data mining for identification of molecular targets in ovarian cancer. Asian Pac J Cancer Prev 17: 1691-1699, 2016.

27. McCall MN, Murakami PN, Lukk M, Huber W and Irizarry RA Assessing affymetrix Genechip microarray quality. BMC Bioinformatics 12: 137, 2011

28. Northcott PA, Korshunov A, Witt H, Hielscher T, Eberhart CG, Mack S, Bouffet E, Clifford SC, Hawkins CE, French P, et al: Medulloblastoma comprises four distinct molecular variants. J Clin Oncol 29: 1408-1414, 2011.

29. Li KK, Lau KM and Ng HK: Signaling pathway and molecular subgroups of medulloblastoma. Int J Clin Exp Pathol 6: 1211-1222, 2013
30. Thompson MC, Fuller C, Hogg TL, Dalton J, Finkelstein D, Lau CC, Chintagumpala M, Adesina A, Ashley DM, Kellie SJ, et al: Genomics identifies medulloblastoma subgroups that are enriched for specific genetic alterations. J Clin Oncol 24: 1924-1931, 2006.

31. Cho YJ, Tsherniak A, Tamayo P, Santagata S, Ligon A, Greulich H, Berhoukim R, Amani V, Goumnerova L, Eberhart CG, et al: Integrative genomic analysis of medulloblastoma identifies a molecular subgroup that drives poor clinical outcome. J Clin Oncol 29: 1424-1430, 2011.

32. Hooper CM, Hawes SM, Kees UR, Gottardo NG and Dallas PB: Gene expression analyses of the spatio-temporal relationships of human medulloblastoma subgroups during early human neurogenesis. PLoS One 9: e112909, 2014.

33. Dittmer S, Kovacs Z, Yuan SH, Siszler G, Kögl M, Summer H, Geerts A, Golz S, Shioda T and Methner A: TOX3 is a neuronal survival factor that induces transcription depending on the presence of CITED1 or phosphorylated CREB in the transcriptionally active complex. J Cell Sci 124 (Pt 2): 252-260, 2011.

34. Yuan SH, Qiu Z and Ghosh A: TOX3 regulates calcium-dependent transcription in neurons. Proc Natl Acad Sci USA 106: 2909-2914, 2009.

35. Tessema M, Yingling CM, Grimes MJ, Thomas CL, Liu Y, Leng S, Joste $\mathrm{N}$ and Belinsky SA: Differential epigenetic regulation of TOX subfamily high mobility group box genes in lung and breast cancers. PLoS One 7: e34850, 2012.

36. Morohashi Y, Hatano N, Ohya S, Takikawa R, Watabiki T, Takasugi N, Imaizumi Y, Tomita T and Iwatsubo T: Molecular cloning and characterization of CALP/KChIP4, a novel EF-hand protein interacting with presenilin 2 and voltage-gated potassium channel subunit Kv4. J Biol Chem 277: 14965-14975, 2002.

37. Northcott PA, Shih DJ, Peacock J, Garzia L, Morrissy AS, Zichner T, Stütz AM, Korshunov A, Reimand J, Schumacher SE, et al: Subgroup-specific structural variation across 1,000 medulloblastoma genomes. Nature 488: 49-56, 2012.

38. Engelender S, Kaminsky Z, Guo X, Sharp AH, Amaravi RK, Kleiderlein JJ, Margolis RL, Troncoso JC, Lanahan AA, Worley PF, et al: Synphilin-1 associates with alpha-synuclein and promotes the formation of cytosolic inclusions. Nat Genet 22: 110-114, 1999.

39. Rudin CM, Hann CL, Laterra J, Yauch RL, Callahan CA, Fu L, Holcomb T, Stinson J, Gould SE, Coleman B, et al: Treatment of medulloblastoma with hedgehog pathway inhibitor GDC-0449. N Engl J Med 361: 1173-1178, 2009.

This work is licensed under a Creative Commons Attribution-NonCommercial-NoDerivatives 4.0 International (CC BY-NC-ND 4.0) License. 